\title{
Predição do peso vivo a partir de medidas corporais em animais mestiços Holandês/Gir
}

\author{
Prediction of live weight based on body measurements in crossbred animals Holstein/Gir
}

\author{
Guilherme Lanna Reis ${ }^{\mathrm{I}}$ Fernando Henrique Melo Andrade Rodrigues Albuquerque ${ }^{\mathrm{I}}$ \\ Bruno Dourado Valente ${ }^{\mathrm{I}^{*}}$ Gabrimar Araújo Martins ${ }^{\mathrm{II}}$ Roberto Luiz Teodoro ${ }^{\mathrm{III}}$ \\ Marcos Brandão Dias Ferreira ${ }^{\mathrm{IV}}$ João Bosco Neves Monteiro ${ }^{\text {III }}$ \\ Martinho de Almeida e Silva ${ }^{\mathrm{I}}$ Fernando Enrique Madalena ${ }^{\mathrm{I}}$
}

\section{RESUMO}

O objetivo deste estudo foi investigar as relações entre o peso corporal e as medidas corporais altura de garupa (ag), comprimento de garupa (cg), comprimento corporal (cc) e perímetro torácico (pt), em bovinos oriundos principalmente do cruzamento das raças Holandês e Gir. Foram utilizados dados de 483 vacas, 469 novilhas e 62 machos, de três rebanhos distintos, analisados separadamente para cada categoria a fim de estabelecer equações polinomiais dos pesos em relação às medidas corporais. As correlações simples do peso corporal com $\mathrm{pt}, \mathrm{cc}, \mathrm{cg}$ e ag foram respectivamente 0,$807 ; 0,440 ; 0,187$ e 0,504 para vacas; 0,$928 ; 0,735 ; 0,819$ e 0,880 machos, e 0,942; 0,748; 0,902 e 0,573 para novilhas. Embora as regressões de peso corporal em relação ao cc e cg tenham sido significativas $(P<0,05), \quad o$ aumento $d a$ porcentagem de explicação das variações do peso corporal obtido com a inclusão destas medidas, em adição ao pt, não parece justificar o custo das medições. As equações de predição do peso corporal em função do pt foram as seguintes: para vacas, peso $=12.174-187,410 \mathrm{pt}+0,97196960 \mathrm{pt}^{2}-$ $0,00162382 \mathrm{pt}^{3}$, para novilhas, peso $=1.717-35,167 \mathrm{pt}+$ $0,238978 p t^{2}-0,00046260 \mathrm{pt}^{3}$ e, para machos, peso = $3.862+76,014 p t-0,488837 p t^{2}+0,00109755 p t^{3}$.

Palavras-chave: animal mestiço, correlação, perimetro torácico, peso corporal, regressão.

\section{ABSTRACT}

The objective of this study was to investigate the relationship between hip height (ag), rump length (cg), body length (cc) and heart girth (pt) with live body weight of crossbred animals, mainly from the cross between Holstein and Gir breeds. Data on 483 cows, 469 heifers and 62 males in three herds were analyzed for each category using polynomial regression equations of body weight on measurements. The correlations between body weight and $\mathrm{pt}, \mathrm{cc}, \mathrm{cg}$ and ag were, respectively $0.807,0.440,0.187$ and 0.504 for cows, 0.928, 0.735, 0.819 and 0.880 for males and 0.942, 0.748, 0.902 and 0.573 for heifers. Although the regressions of body weights on $\mathrm{cc}$ and $\mathrm{cg}$ were significant $(P<0.05)$, the additional goodness of fit of a model that includes these two traits in addition to heart girth does not justify the extra cost for recording these traits. The prediction equations were: for cows, body weight $=12174$ $187.410 p t+0.97196960 \mathrm{pt}^{2}-0.00162382 \mathrm{pt}^{3}$, for heifers, body weight $=1717-35.167 p t+0.238978 p t^{2}-0.00046260 p t^{3}$ and for males, body weight $=-3862+76.014 p t$ $0.488837 p t^{2}+0.00109755 p t^{3}$.

Key words: crossbred animal, correlation, heart girth, live weight, regression.

\section{INTRODUÇÃO}

A determinação do peso corporal dos animais é importante para avaliar o crescimento e o estado nutricional, administrar adequadamente remédios e parasiticidas, estabelecer o valor de venda do animal de corte e ajustar o arraçoamento. Entretanto, a realidade econômica das fazendas leiteiras do Brasil muitas vezes não permite a aquisição de balanças para realizar a pesagem dos animais. Para minimizar tal deficiência, pode ser utilizada a alternativa de se predizer o peso por meio de algumas medidas corporais.

'Universidade Federal de Minas Gerais (UFMG), Departamento de Zootecnia, Escola de Veterinária. Av. Antônio Carlos, 6627, CP 567, 30123-970, Belo Horizonte, MG, Brasil. E-mail: bvalente66@yahoo.com.br.*Autor para correspondência.

"Universidade Estadual Vale do Acarau, Sobral, CE, Brasil.

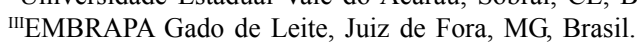

${ }^{\mathrm{IV}}$ EPAMIG, Fazenda Experimental Santa Rita, Prudente de Morais, MG, Brasil 
As medidas corporais mais mencionadas na literatura para predizer o peso são o perímetro torácico, o comprimento corporal, a altura da cernelha e da garupa e o comprimento da garupa. Embora existam discrepâncias sobre qual medida individual deve ser utilizada para predizer o peso, a acurácia da predição tem sido geralmente alta especialmente quando mais de uma medida for considerada (HEIRINCHS et al., 1992; KHALIL e VACCARO, 2002).

As regressões do peso sobre as medidas corporais e a acurácia da estimação podem ser influenciadas pela raça, idade, condição corporal e estado fisiológico do animal (HEINRICHS et al., 1992), o que justifica o exame da conveniência que utiliza equações de predição diferentes para cada classe.

Atualmente há no mercado brasileiro fitas que estimam o peso com base no perímetro torácico, mas que foram confeccionadas segundo medidas $\mathrm{e}$ pesos de animais leiteiros Bos taurus e que poderiam ser aprimoradas com dados obtidos nas condições locais.

O objetivo deste estudo foi investigar as relações entre as medidas altura e comprimento de garupa, comprimento corporal e perímetro torácico e o peso corporal em bovinos oriundos do cruzamento entre as raças Holandês e Gir.

\section{MATERIAL E MÉTODOS}

Foram analisados pesos e medidas corporais de 1.014 animais mestiços Holandês/Gir, avaliados entre abril e julho de 2002. Os animais foram agrupados em três categorias (vacas, novilhas e machos), e são provenientes de três fazendas experimentais: Campo Experimental de Coronel Pacheco, no município de Coronel Pacheco, MG e Campo Experimental Santa Mônica, no município de Valença, RJ, ambos pertencentes à Embrapa Gado de Leite e Fazenda Experimental Santa Rita - EPAMIG, no município de Sete Lagoas, MG. Os números de observações e as idades, para cada categoria, são apresentados na tabela 1 .

Os animais apresentaram frações de genes de raça européia de $1 / 4,3 / 8,1 / 2,5 / 8,3 / 4,7 / 8$ e 8/8 nos

Tabela 1 - Número de observações $(\mathrm{N})$ e idades (em meses), por categoria.

\begin{tabular}{llccc}
\hline & & \multicolumn{3}{c}{ Idade } \\
\cline { 3 - 5 } Categoria & $\mathrm{N}$ & Média & Mínima & Máxima \\
\hline Vacas & 483 & 47 & 29 & 163 \\
Novilhas & 469 & 25 & 7 & 48 \\
Machos & 62 & 19 & 8 & 60 \\
\hline
\end{tabular}

seguintes percentuais respectivamente: $0,3,7,5,33,0$, $2,3,16,533,4$ e $4 \%$ (3\% desconhecido). Os pais eram da raça Holandesa $(61,7 \%)$, Gir $(12,2 \%)$, mestiços $(23,4 \%)$ e desconhecido $(2,6 \%)$ e as mães das raças Holandesa (1,1\%), Gir (18,8\%), mestiços Holandês/Gir (76,8\%) e Guzerá $(3,3 \%)$.

As características medidas foram: o perímetro torácico $(\mathrm{pt}=$ perímetro imediatamente caudal à escápula passando pelo esterno e pelos processos espinhais das vértebras torácicas), comprimento corporal ( $\mathrm{cc}=$ linha reta entre a articulação escápuloumeral e a tuberosidade coxal do ílio), comprimento da garupa ( $\mathrm{cg}=$ linha reta entre a tuberosidade coxal do ílio e a tuberosidade isquiática) e altura da garupa (ag=distância entre o solo e a tuberosidade sacral do ílio com os animais em superfície plana). Aúltima medida é mencionada por LISBOA \& FERNANDES (1987) como mais precisa que a altura à cernelha, pois, em razão do posicionamento do animal, apresenta menor variação no momento da medição. O pt foi medido com fita métrica, o cg e o cc com trena, e a ag com bastão barimétrico. Os dados de vacas, novilhas e machos de recria foram analisados separadamente. Nas vacas, foram considerados três estádios fisiológicos: em lactação, seca e na maternidade (um mês pré-parto estimado) e três grupos genéticos, segundo a proporção de Bos taurus: 1) de 0,375 a 0,625,2) de 0,625 a 0,75 e 3 ) de 0,75 a 0,968 .

Para as vacas, foram realizadas análises de variância do peso utilizando-se o Proc GLM do pacote SAS (1998) segundo o seguinte modelo estatístico: $y_{i j k l m}=\mu+\mathrm{F}_{\mathrm{i}}+\mathrm{M}_{\mathrm{ij}}+\mathrm{G}_{\mathrm{ik}}+\mathrm{E}_{\mathrm{il}}+\mathrm{pt}+\mathrm{cc}+\mathrm{cg}+\mathrm{ag}+\mathrm{Gpt}_{i k}$ ${\mathrm{G} c c_{i k}}^{i j k l \mathrm{Gcg}_{i k}}+\mathrm{G}^{\mathrm{H}} a g_{i k}+\mathrm{eij}_{k l m}$, em que $\mathrm{y}_{\mathrm{ijklm}}$ é o peso da vaca $\mathrm{m}$ de grau de sangue $\mathrm{k}$ em estádio físiológico $1 \mathrm{na}$ fazenda i coletada no mês $\mathrm{j}, \mu$ é a média populacional, $\mathrm{F}_{\mathrm{i}}$ é o efeito da fazenda i, M é o efeito do mês de coleta $j$ aninhado à fazenda $i, G_{i k}{ }_{i j}$ é o efeito do grupo genético $k$ aninhado à fazenda $i, E_{i l}$ é o efeito do estádio fisiológico $l$ aninhado à fazenda $i$. As medidas $p t, c c$, $c g$, e $a g$ são covariáveis lineares relativas às medidas corporais, $G p t_{i k}, G c c_{i k}, G c g_{i k}$ e $G a g_{i k}$ são as interações entre as covariáveis lineares e o grupo genético $k$ aninhado à fazenda $i$ e $e_{i j k l m}$ é o resíduo associado à observação $y_{i j k l m}$. As interações consideram a variação dos efeitos lineares das medidas corporais sobre o peso em diferentes combinações de grupos genéticos e fazendas.

Também foram estudados, para as três categorias, modelos de regressão cúbica considerando como variáveis explicativas apenas as quatro medidas e desconsiderando todas as variáveis classificatórias. Para isso foi utilizado o procedimento de eliminação "passo a passo" (opção backwards) do Proc REG do 
pacote SAS (1998). A aderência do modelo foi avaliada por meio do coeficiente de determinação, ajustado pelos graus de liberdade do modelo $\mathrm{Aj} . \mathrm{R}^{2}$ (INSTAT, 2002).

\section{RESULTADOS E DISCUSSÃO}

As correlações simples entre as medidas corporais e o peso corporal em vacas, machos e novilhas estão representadas na tabela 2 . Medidas corporais de machos e novilhas, de maneira geral, apresentaram altas correlações com o peso. Em contrapartida, em vacas, apenas o pt apresentou maior grau de associação com o peso corporal. Para todas as categorias, assim como observado por HEINRICHS et al. (1992), o pt foi a variável que apresentou maior correlação com o peso.

$\mathrm{Na}$ tabela 3, são apresentados os coeficientes de determinação, ajustados pelos graus de liberdade, para diferentes modelos, incluindo apenas coeficientes de regressão do peso em função das medidas corporais das vacas. Considerando o modelo utilizado na análise de variância, que inclui todos os efeitos de classificação, o coeficiente de determinação ajustado foi 0,762 . Retirando-se do modelo as interações das covariáveis lineares das quatro medidas por grupo genético/fazenda, o coeficiente de determinação ajustado foi 0,749 . Para o modelo que inclui apenas coeficientes de regressão relativos às medidas, o coeficiente de determinação ajustado obtido foi 0,733 . Desse modo, a remoção dos efeitos classificatórios e das interações apresenta queda de pouca importância na qualidade de aderência do modelo. Tal aspecto torna exeqüível a predição do peso dos animais em função apenas de medidas corporais, uma vez que informações como o grau de sangue nem sempre são disponíveis.

Quando se excluiu o pt, o $\mathrm{R}^{2}$ ajustado reduziu para 0,412 . Separadamente, o pt, como variável explicativa do peso, apresentou $\mathrm{R}^{2}$ ajustado de 0,663 . Tanto as correlações quanto os coeficientes de determinação encontrados estão dentro da amplitude de variação mencionada na literatura, de 0,70 a 0,99 (GUARAGNA et al., 1993; POONIA e RAO, 1999) e de 0,62 a 0,94 (MORALES e MONTES DE OCA, 1994; KOENEN e GROEN, 1998), respectivamente.

Os coeficientes de regressão relativos à ag não apresentaram efeito significativo no modelo que inclui as quatro medidas. Embora os coeficientes de regressão do peso corporal em relação ao $\mathrm{cc}$ e $\mathrm{cg}$ fossem significativos, o aumento da aderência do modelo pela inclusão destas variáveis explicativas não parece justificar o custo adicional de sua medição. HEINRICHS et al. (1992) também observaram que seria de pouco valor a adição de segunda medida para predizer o peso corporal.

Com exceção de HEINRICHS et al. (1992) e GUARAGNA et al. (1993), nenhum dos autores

Tabela 2 - Estatísticas descritivas e correlações (Pearson) de peso e medidas corporais, perímetro torácico (pt), altura da garupa (ag), comprimento corporal (cc) e comprimento da garupa (cg), em vacas, machos e novilhas.

\begin{tabular}{|c|c|c|c|c|c|c|c|}
\hline \multirow{2}{*}{ Categoria } & \multirow{2}{*}{ Variáveis } & \multirow{2}{*}{ Média $(\mathrm{kg} / \mathrm{cm})$} & \multirow{2}{*}{ Desvio padrão } & \multicolumn{4}{|c|}{ 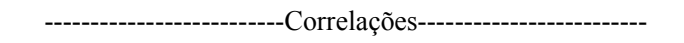 } \\
\hline & & & & pt & $\mathrm{cc}$ & $\mathrm{ag}$ & $\operatorname{cg}$ \\
\hline \multirow{5}{*}{ Vacas $^{1}$} & peso $(\mathrm{kg})$ & 512,09 & 67,01 & 0,807 & 0,440 & 0,504 & 0,187 \\
\hline & $\mathrm{pt}(\mathrm{cm})$ & 188,39 & 9,53 & & 0,334 & 0,490 & 0,441 \\
\hline & $\mathrm{cc}(\mathrm{cm})$ & 112,59 & 5,41 & & & 0,359 & 0,018 \\
\hline & ag $(\mathrm{cm})$ & 137,86 & 5,86 & & & & $-0,033$ \\
\hline & $\mathrm{cg}(\mathrm{cm})$ & 45,3 & 5,33 & & & & \\
\hline \multirow{5}{*}{ Machos $^{2}$} & peso $(\mathrm{kg})$ & 250,76 & 96,49 & 0,928 & 0,735 & 0,880 & 0,819 \\
\hline & $\mathrm{pt}(\mathrm{cm})$ & 150,05 & 19,61 & & 0,772 & 0,915 & 0,784 \\
\hline & $\mathrm{cc}(\mathrm{cm})$ & 92,26 & 12,31 & & & 0,758 & 0,781 \\
\hline & ag $(\mathrm{cm})$ & 124,52 & 10,77 & & & & 0,793 \\
\hline & $\operatorname{cg}(\mathrm{cm})$ & 39,06 & 5,23 & & & & \\
\hline \multirow{5}{*}{ Novilhas $^{3}$} & peso ( $\mathrm{kg}$ ) & 335,70 & 109,49 & 0,942 & 0,748 & 0,573 & 0,902 \\
\hline & $\mathrm{pt}(\mathrm{cm})$ & 162,66 & 19,37 & & 0,765 & 0,627 & 0,910 \\
\hline & $\mathrm{cc}(\mathrm{cm})$ & 98,80 & 10,82 & & & 0,578 & 0,748 \\
\hline & $\mathrm{ag}(\mathrm{cm})$ & 39,81 & 5,40 & & & & 0,585 \\
\hline & $\mathrm{cg}(\mathrm{cm})$ & 128,89 & 9,50 & & & & \\
\hline
\end{tabular}

${ }^{1}$ Prob $<0,0001$ para correlações $>0,33 .{ }^{2}$ Prob $<0,0001$ para correlações $>0,73 .{ }^{3}$ Prob $<0,0001$ para correlações $>0,57$.

Ciência Rural, v.38, n.3, mai-jun, 2008. 
Tabela 3 - Coeficientes de regressão do peso em função das medidas corporais, perímetro torácico (pt), altura da garupa (ag), comprimento corporal(cc) e comprimento da garupa (cg) para modelos obtidos pela eliminação "passo a passo" (Backwards) e com maior $\mathrm{R}^{2}$ ajustado após eliminação de uma, duas e três variáveis explicativas, em vacas.

\begin{tabular}{|c|c|c|c|c|}
\hline & \multicolumn{4}{|c|}{ Variáveis consideradas } \\
\hline & Backwards & $\mathrm{cc} c \mathrm{pt}$ & pt cg & $\mathrm{pt}$ \\
\hline Intercepto & 10033 & 10033 & 9685 & 12174 \\
\hline pt & $-209,784$ & $-209,784$ & $-208,183$ & $-187,410$ \\
\hline \multicolumn{5}{|l|}{$\mathrm{cc}$} \\
\hline $\mathrm{cg}$ & 239,487 & 239,487 & 257,153 & \\
\hline \multicolumn{5}{|l|}{ ag } \\
\hline $\mathrm{pt}^{2}$ & 1,087731 & 1,087732 & 1,083177 & 0,971969 \\
\hline \multicolumn{5}{|l|}{$\mathrm{cc}^{2}$} \\
\hline $\mathrm{cg}^{2}$ & $-5,360778$ & $-5,360778$ & $-5,766164$ & \\
\hline \multicolumn{5}{|l|}{$\mathrm{ag}^{2}$} \\
\hline $\mathrm{pt}^{3}$ & $-0,00182289$ & $-0,00182289$ & $-0,00181754$ & $-0,00162382$ \\
\hline $\mathrm{cc}^{3}$ & 0,00005328 & 0,00005328 & & \\
\hline $\operatorname{cg}^{3}$ & 0,03931974 & 0,03931974 & 0,04234174 & \\
\hline \multicolumn{5}{|l|}{$\mathrm{ag}^{3}$} \\
\hline $\mathrm{R}^{2}$ ajustado & 0,733 & 0,733 & 0,710 & 0,663 \\
\hline
\end{tabular}

consultados na literatura utilizou regressões cúbicas, mas apenas regressões lineares e quadráticas. Entretanto, neste estudo, os modelos testados sempre apresentaram efeitos cúbicos significativos, exceto o que inclui apenas altura da garupa isoladamente.

No modelo selecionado pelo procedimento "passo a passo" para a categoria de machos $\left(\mathrm{R}^{2}\right.$ ajustado $=0,963)$, foram significativos os termos cúbicos de todas as medidas com exceção de $\mathrm{ag}^{3}$ (Tabela 4). O R ${ }^{2}$ ajustado, 0,926, obtido com a inclusão única do pt, é semelhante ao do modelo obtido pela eliminação "passo a passo", o que justifica a utilização apenas desta medida para predizer o peso.

Resultados semelhantes foram obtidos para a categoria novilhas. $\mathrm{O}$ modelo de predição com base apenas no pt apresentou aderência ligeiramente menor do que a obtida com base nas quatro medidas (Tabela 5).

As relações entre o peso e o perímetro torácico estão representadas graficamente para as três categorias estudadas na figura 1. A obtenção de curvas

Tabela 4 - Coeficientes de regressão do peso em função das medidas corporais, perímetro torácico (pt), altura da garupa (ag), comprimento corporal (cc) e comprimento da garupa (cg) para modelos obtidos pela eliminação "passo a passo" (Backwards) e com maior $\mathrm{R}^{2}$ ajustado após eliminação de uma, duas e três variáveis explicativas, em machos.

\begin{tabular}{|c|c|c|c|c|}
\hline & \multicolumn{4}{|c|}{ Variáveis consideradas } \\
\hline & Backwards & ag .cg. pt & cg. pt & $\mathrm{pt}$ \\
\hline Intercepto & -3087 & -3209 & -3052 & -3862 \\
\hline pt & 61,116 & 64,150 & 61,397 & 76,014 \\
\hline \multicolumn{5}{|l|}{$\mathrm{cc}$} \\
\hline \multicolumn{5}{|l|}{$\mathrm{cg}$} \\
\hline \multicolumn{5}{|l|}{ ag } \\
\hline $\mathrm{pt}^{2}$ & $-0,401236$ & $-0,410713$ & $-0,378008$ & $-0,488837$ \\
\hline $\mathrm{cc}^{2}$ & 0,037608 & & & \\
\hline $\operatorname{cg}^{2}$ & $-0,281087$ & $-0,265981$ & $-0,37747096$ & \\
\hline $\mathrm{ag}^{2}$ & 0,008653 & 0,007967 & & \\
\hline $\mathrm{pt}^{3}$ & 0,00089863 & 0,00090321 & 0,00081172 & 0,00109755 \\
\hline $\mathrm{cc}^{3}$ & $-0,00022577$ & & & \\
\hline $\operatorname{cg}^{3}$ & 0,00516317 & 0,00506423 & 0,00712265 & \\
\hline \multicolumn{5}{|l|}{$\mathrm{ag}^{3}$} \\
\hline $\mathrm{R}^{2}$ ajustado & 0,963 & 0,955 & 0,953 & 0,926 \\
\hline
\end{tabular}

Ciência Rural, v.38, n.3, mai-jun, 2008. 
Tabela 5 - Coeficientes de regressão do peso em função das medidas corporais, perímetro torácico (pt), altura da garupa (ag), comprimento corporal (cc) e comprimento da garupa (cg) para modelos obtidos pela eliminação "passo a passo" (Backwards) e com maior $\mathrm{R}^{2}$ ajustado após eliminação de uma, duas e três variáveis explicativas, em novilhas.

\begin{tabular}{llll}
\hline & & \multicolumn{2}{c}{ Variáveis consideradas } \\
\cline { 2 - 4 } & Backwards & $\mathrm{cc} \cdot \mathrm{cg} \cdot \mathrm{pt}$ & $\mathrm{cc} \cdot \mathrm{pt}$ \\
\hline Intercepto & 8425 & 1904 & 1882 \\
$\mathrm{pt}$ & $-5,933$ & $-38,516$ & $-38,005$ \\
$\mathrm{cc}$ & & & $-35,167$ \\
$\mathrm{cg}$ & $-0,808$ & & 0,240984 \\
$\mathrm{ag}$ & $-196,574$ & & 0,030699 \\
$\mathrm{pt}$ & 0,029520 & 0,244909 & 0,238978 \\
$\mathrm{cc}^{2}$ & 0,024179 & 0,032574 & $-0,00044554$ \\
$\mathrm{cg}^{2}$ & & $-0,010522$ & $-0,00013598$ \\
$\mathrm{ag}^{2}$ & 1,573398 & & $-0,0004626$ \\
$\mathrm{pt}^{3}$ & & $-0,0045405$ & 0,902 \\
$\mathrm{cc}^{3}$ & $-0,00010525$ & $-0,00014385$ & \\
$\mathrm{cg}^{3}$ & & & 0,894 \\
$\mathrm{ag}^{3}$ & $-0,00411892$ & 0,903 & \\
$\mathrm{R}^{2}$ ajustado & 0,913 & & \\
\hline
\end{tabular}

diferentes demonstra que a relação entre as duas variáveis, peso e pt, não é a mesma em categorias distintas. Dessa maneira, torna-se necessária a utilização de modelos de regressão específicos para a predição de peso em função do perímetro torácico em cada categoria.

\section{CONCLUSÕES}

O peso pode ser estimado por meio de modelo que inclui medidas corporais, sendo o pt a variável explicativa que mais contribui com a aderência do modelo.

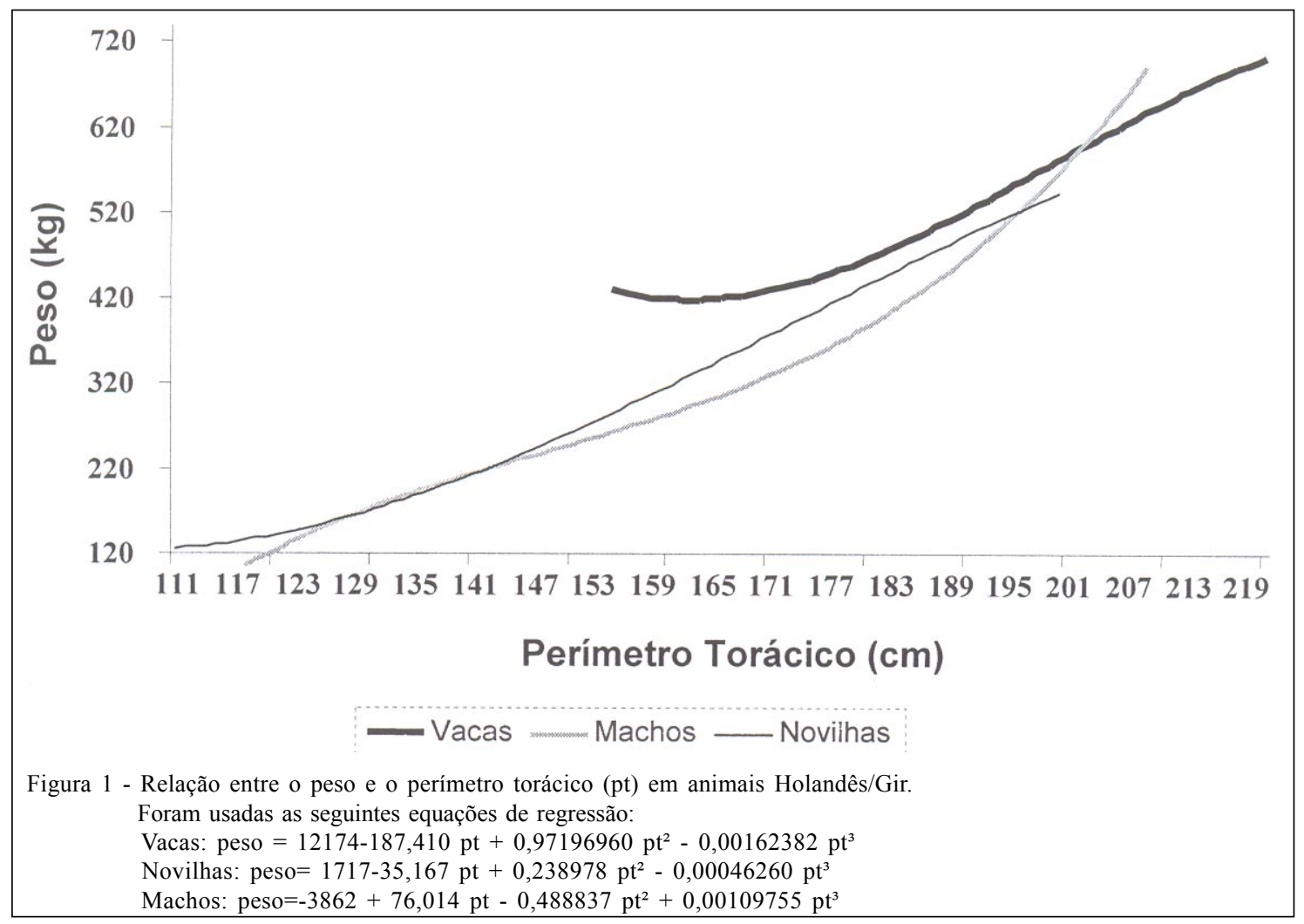

Ciência Rural, v.38, n.3, mai-jun, 2008. 
Modelos polinomiais para predição do peso com base no perímetro torácico, quando incluído até o termo cúbico, apresentaram alta aderência e foram distintos para as categorias vacas, novilhas e machos.

\section{REFERÊNCIAS}

GUARAGNA, G.P. et al. Relationships between body weight and measurements in Mantiqueira dairy heifers. Boletim de Indústria Animal, v.50, p.101-106, 1993.

HEINRICHS, A.J. et al. Predicting body weight and wither height in Holstein heifers using body measurements. Journal of Dairy Science, v.75, p.3576-3581, 1992.

INSTAT. The instat guide to choosing and interpreting statistical tests. [s.n.]:[s.n.], 2002. Acesso em 20/10/2002. Online. Disponível em: <www.graphpad.com>.

KHALIL, R.; VACCARO, L. Body weights and measurements in dual purpose cows: their interrelation and association with genetic merit for three production traits. Zootecnia Tropical, v. 20, p. $11-30,2002$.

KOENEN, E.P.C.; GROEN, A.F. Genetic evaluation of body weight of lactating Holstein heifers using body measurements and conformation traits. Journal of Dairy Science, v.81, p.1709-1713, 1998.

LISBOA, S.R.; FERNANDES, L.C.O. Efeito do tamanho corporal na fertilidade da primeira e segunda estação de monta, e na produtividade de fêmeas cruza Charolês. Revista da Sociedade Brasileira de Zootecnia, v.16, p.204-214, 1987.

MORALES, R.C.; DE OCA, R. V. M. Estimación del peso vivo por el perímetro torácico y/o la altura de la cruz en hembras bovinas lecheras del cruce Holstein-Cebú. Revista de la Producción Animal, v.8, n.2, p.179-182, 1994.

POONIA, R.S.; RAO, R. Growth in Haryana and crossbred (Holstein-Friesan x Haryana) calves. International Journal Animal Science, v.14, p.97-99, 1999.

SAS. USER'S Guide. 4.ed. Cary: SAS Institute, 1998. 842p. 